\title{
Marion Coville, Noémie Couillard et Karin Schlageter (dir.) : Les coulisses du musée
}

\section{Michèle Gellereau}

\section{(2) OpenEdition}

\section{Journals}

Édition électronique

URL : http://journals.openedition.org/edc/6857

DOI : $10.4000 /$ edc.6857

ISSN : 2101-0366

Éditeur

Université de Lille

Édition imprimée

Date de publication : 1 juin 2017

Pagination : 207-210

ISBN : 978-2-917562-17-8

ISSN : $1270-6841$

Référence électronique

Michèle Gellereau, « Marion Coville, Noémie Couillard et Karin Schlageter (dir.) : Les coulisses du musée », Études de communication [En ligne], 48 | 2017, mis en ligne le 01 juin 2017, consulté le 23 septembre 2020. URL : http://journals.openedition.org/edc/6857 ; DOI : https://doi.org/10.4000/edc.6857

Ce document a été généré automatiquement le 23 septembre 2020.

(c) Tous droits réservés 


\title{
Marion Coville, Noémie Couillard et Karin Schlageter (dir.) : Les coulisses du musée
}

\author{
Michèle Gellereau
}

\section{RÉFÉRENCE}

Coville M., Couillard N. et Schlageter K. (dir.) (2016). Les coulisses du musée, revue POLI (Politique de l'image) nº 12, Paris, POLI Editions.

1 Ce numéro 12 de la revue POLI se veut original, en harmonie avec son titre et de fait, il nous propose d'explorer ce qui, dans l'espace muséal, est rarement étudié : "les pratiques quotidiennes des professionne-l-le-s restées dans l'ombre en raison de leur caractère usuel et routinier " ( $4^{\mathrm{e}}$ de couverture). Mais il nous fait découvrir plus que des coulisses : une manière de voir et de faire le musée qui engage le lecteur dans une expérience esthétique autant que dans un travail intellectuel de lecture critique. En effet, son contenu alterne des types de textes variés: articles de recherche, témoignages, entretiens, textes poétiques, photographies. Par ailleurs, il se structure autour de différentes questions (l'exposition, les médiations, la régie, les pratiques, les sites web...) non seulement du point de vue des chercheurs mais aussi du point de vue des acteurs, des artistes, des personnels et d'autres " professionnel(e)s».

Ce sont par ailleurs des expériences et recherches de différents pays (France, Italie, Suisse, Allemagne, États-Unis, Australie...) qui nourrissent ici des approches multidisciplinaires en sciences humaines et sociales.

2 Les trois directrices de cet ouvrage (Marion Coville, Noémie Couillard et Karin Schlageter) donnent tout de suite le ton en proposant une présentation titrée «Entrée réservée au personnel » (p.6). Avant de procéder à la présentation thématisée des textes, cette ouverture met l'accent sur la nécessité de ne pas masquer la multiplicité des voix et des acteurs qui font l'activité muséale par la « voix unique et officielle » de 
l'institution (p. 7). Sans être pour autant un membre du "personnel», le lecteur pénètre facilement dans cette entrée qui propose d'emblée de solides jalons théoriques : les travaux de l'anthropologue Sharon Mac Donald, d'Howard Becker et de plusieurs chercheurs français qui ont écrit sur les professions du musée. Le lecteur est invité dans cette introduction à s'emparer des questions à partir de trois entrées : les approches critiques du musée, les politiques de la médiation, les corps et les objets.

Pourtant, la structure de l'ouvrage n'est pas organisée par rapport à ces thèmes et finalement, chaque article fonctionne de manière indépendante et ne semble pas forcément s'intégrer dans une seule thématique. Certains articles dépassent largement le thème du numéro. Ainsi le long article ( 22 pages) de Tony Bennet, traduit de l'anglais "Le dispositif expositionnaire " (p.14) est très intéressant et propose une approche critique de l'institution muséale se fondant sur Foucault. Connu pour son enrichissement des approches du musée dans une perspective "cultural studies", Bennet livre ici une réflexion en partie historique sur le dispositif expositionnaire qui engage un processus social de régulation. Qu'apprend le citoyen en allant au musée ? Sans doute beaucoup, mais surtout, selon Bennet, à adhérer aux valeurs, organisations des connaissances et perspectives des pouvoirs en place. En s'intéressant à certaines grandes expositions (Expositions universelles de 1851, de 1889 entre autres), il fait tomber le masque du musée ou de l'exposition comme lieu d'éducation citoyenne ahistorique et a-politique.

Débordant également le cadre du musée pour s'intéresser aux visites de sites patrimoniaux, la contribution de Sharon Mac Donald (également traduite de l'anglais) : «La médiation du patrimoine : les guides touristiques de l'ancien site des congrès du parti nazi à Nuremberg " (p.50) est passionnante dans son approche politique de la médiation et de la visite guidée. En examinant comment les guides d'un site aussi sensible (désigné par l'auteure comme "difficult heritage» ou "patrimoine embarrassant » dans la traduction) encouragent des lectures préférentielles du site tout en essayant d'être objectifs, elle entre dans le cœur de la question de l'impossible neutralité de la médiation. C'est donc là aussi une question qui dépasse largement la question du musée et celle de ses coulisses. Ces deux articles par ailleurs ancrés dans l'étude concrète de lieux révèlent bien des aspects des institutions muséales et patrimoniales, ainsi de la difficulté à interpréter les lieux quand on évite de saisir leur portée politique. Ils sont exemplaires de la richesse théorique et critique de ce numéro de revue qui à la fois cerne précisément son sujet et va plus loin en proposant de remettre en cause une vision idéalisée de l'éducation muséale.

5 Le lecteur se trouve par ailleurs confronté à un autre type d'approche critique de l'exposition, celle de l'artiste qui, comme jadis Duchamp, remet en cause art et expositions, dans le "portfolio » très imagé de Karin Schlageter « A museum is a museum » (p. 38). Ces photographies d'installations et le court texte qui les précède ancrent le lecteur dans le vécu du musée par l'artiste et dans le choc esthétique. C'est un peu le même trouble ou enchantement qui semble opérer à la lecture des textes et des cartels de Sam Cambio (p. 76) qui offre une perspective de la fonction de gardien de musée inhabituelle et passionnante. On a l'impression que ces "travaux pratiques" qui ponctuent les approches universitaires en disent au moins autant si ce n'est plus que les textes, de par leur ouverture à l'imaginaire du lecteur. D'autres articles incitent ce dernier à élargir son point de vue, à déstructurer ses apriori en lui proposant de comprendre des professions et activités peu explorées dans la littérature académique : 
l'entretien de Noémie Couillard avec Amélie Matray (régisseuse), "La régie des œuvres : anticipation, matérialité des œuvres et éthique professionnelle » (p. 90) et la contribution de Marc-Olivier Gonseth "Archiver des châteaux de sable» (p.96) montrent combien les questions de documentation, catalogage, de matérialité des objets sont fondamentales au musée. Les choix qui sont effectués décrivent le travail d'équipe qui existe dans les musées ou, pour reprendre une référence de Becker, les chaînes de coopération souvent invisibles aux publics et qui pourtant construisent les savoirs et les médiations aboutissant à l'exposition. Mais les musées ne font pas qu'exposer et la contribution de Tiziana Nicoletta Beltrame "Récit insolite des pratiques muséales: ethnographie d'un déploiement des collections»(p.104) et l'entretien avec Ben Fino-Radin, réalisé par Marion Coville et Michael Lo Jacono « Une chasse aux papillons, la conservation des jeux vidéos au MoMa» (p.114) montrent toute la complexité des pratiques d'acquisition, de gestion et de conservation des collections. Car les nouveaux « objets ", les nouvelles procédures liées à la numérisation constituent un patrimoine qui est loin d'être virtuel et exigent une réflexion approfondie sur la pérennité des collections.

Dans une perspective plus ouverte sur le rapport aux publics, l'article de Noémie Couillard sur «La page Facebook d'un établissement patrimonial: entre pêche aux publics et chasses gardées » (p.60) montre d'ailleurs que ces mondes muséaux ne sont pas sans tension et que les conflits de définitions de champs professionnels et d'ouverture aux activités des publics se développent avec les nouveaux médias. Que devient la médiation culturelle et comment aborder désormais les publics? Cette même question est saisie par Maylis Nouvellon dans « Le musée autrement : catégorisation des publics et usages du musée » (p. 68) en s'intéressant aux frontières que certains musées dessinent entre des publics qui seraient ciblés selon des catégories discutables.

Cet ouvrage ne se contente donc pas de mener une réflexion sur les «coulisses du musée », il en montre également toute la richesse, les tensions et les exigences dans l'évolution des techniques. Finalement, ces coulisses ne sont-elles pas tout autant des bases, des cadres d'expériences, des politiques de mises en public au fondement du musée ? Même s'il peut paraître un peu éclaté dans ses thèmes, un des grands intérêts de cet ouvrage est de croiser sous diverses formes les expériences des chercheurs, des publics, des artistes et des spécialistes qui, ensemble, font le musée. La lecture de cette revue bien documentée, facile d'accès et très plaisante à lire peut se faire au gré des centres d'intérêt; elle est en tous cas indispensable pour tous ceux qui souhaitent comprendre le monde muséal et le saisir dans des approches diversifiées.

\section{AUTEUR}

\section{MICHĖLE GELLEREAU}

Univ. Lille, EA 4073 - GERiiCO - Groupement d'Études et de Recherche Interdisciplinaire en

Information et Communication, F-59000 Lille, France

michele.gellereau@univ-lille3.fr 